\title{
Multipolarization Reconfigurable Air-Filled Substrate Integrated Waveguide Cavity-Backed Slot Antenna
}

\author{
Kamil Yavuz Kapusuz, Student Member, IEEE, Sam Lemey, Member, IEEE, \\ and Hendrik Rogier, Senior Member, IEEE
}

\begin{abstract}
A novel single-feed ultra-wideband cavity-backed slot antenna with reconfigurable polarization capability is demonstrated in substrate-independent air-filled substrate integrated waveguide (AFSIW) technology for Internet of Things (IoT) applications in the [5.15-5.85] $\mathrm{GHz}$ frequency band of the unlicensed national information infrastructure (U-NII). The antenna achieves an ultrawide impedance bandwidth by exploiting a double-ring of slots and supports four switchable linear polarization states $\left(-45^{\circ}\right.$, $0^{\circ},+45^{\circ}$ and $90^{\circ}$ ) by leveraging four quartets of PIN diodes. By electrically shorting the slots at well-defined positions by these PIN diodes, dynamic polarization reconfiguration is achieved by switching their DC bias current, supplied at the antenna feed through an external bias tee and routed from the antenna's back to front cavity plane via the AFSIW sidewalls. This new simple, yet effective, bias network enables the integration of all polarization control electronics inside the antenna cavity to protect them from environmental effects. Finally, measurements prove that the prototype exhibits an overlapping impedance bandwidth of $29 \%$, from $4.85 \mathrm{GHz}$ to $6.45 \mathrm{GHz}$, and a stable conically-shaped radiation pattern across the operating bandwidth with a 3-dB beamwidth of $45^{\circ}$ and a peak gain of $6.5 \mathrm{dBi}$ for all four states.
\end{abstract}

Index Terms-Air-filled substrate integrated waveguide (AFSIW), cavity-backed slot antenna, multipolarization reconfigurable, PIN diodes, reconfigurable antennas.

\section{INTRODUCTION}

$\mathbf{T}$ $1 \mathrm{O}$ unleash the full potential of the Internet of Things (IoT) paradigm, uniquely identifiable connected everyday devices with multi-functional services are essential to implement fast, secure and reliable wireless communication in realistic deployment scenarios [1]. To facilitate invisible integration into such devices, without significantly increasing their cost [2], there is a stringent need for low-cost and lowprofile antennas with high isolation between the antenna and its integration platform [3]. For the next generation high-data-rate wireless communications [4], these antennas should guarantee high radiation efficiency and stable ultra-wideband performance with minimal detuning due to varying environmental conditions. Moreover, for smart surfaces, such as smart floors, ceilings or desks, a conical beam pattern, with a null at zenith and peaking at an intermediate elevation angle, should be targeted [5]-[7].

The integration of dynamic polarization reconfiguration in a single-feed air-filled substrate-integrated-waveguide (AFSIW)

Manuscript received December 5, 2020; revised January 30, 2021; accepted Date of publication; date of current version. This work was supported in part by the European Research Council grant 695495 "ATTO: A new concept for ultra-high capacity wireless networks".

The authors are with the Electromagnetics Group, Department of Information Technology, IDLab, Ghent University-IMEC, Technologiepark Zwijnaarde 126, Ghent B-9052, Belgium. (e-mail: kamilyavuz.kapusuz@ugent.be; sam.lemey@ugent.be; hendrik.rogier@ugent.be). antenna is a promising strategy to implement efficient, wideband, and reliable wireless communication in a compact footprint while maintaining a high isolation between the antenna and its platform [8]. In addition, multiple low-cost implementations were proposed that switch between two circular polarizations (CP) [9]-[16], two orthogonal linear polarizations (LP) [17]-[19] and a linear to circular polarization (LP/CP) [20]-[23]. Yet, CP reconfigurable antennas receive any linearly polarized wave with a maximum efficiency of $50 \%$ [24]. In contrast, multilinear polarization reconfigurable antennas significantly improve the system performance in this case, since the polarization efficiency always exceeds $85 \%$ for a reconfigurable antenna switching between four linear polarizations [25].

Relatively few multi-linear polarization-reconfigurable antennas have been proposed, mainly focusing on reconfigurable radiating elements [24]-[31] and feeding networks [32]-[37]. The latter typically suffer from significant feeding network loss, large size and complexity proportional to the number of switching states [33], [34]. Moreover, utmost care is needed to prevent electromagnetic interference (EMI) issues, due to unwanted leakage [35], [36]. As a solution, a reconfigurable radiating element can reduce the feeding network loss, system size and complexity, but after integration into everyday objects its performance is degraded and bandwidth reduced [24]-[31].

This letter proposes a novel [4.85-6.45] GHz-ultra-wideband multilinear polarization reconfigurable AFSIW cavity backedslot antenna, covering all unlicensed national information infrastructure (U-NII) [5.15-5.85] GHz radio bands. This design features (i) four switchable linear polarizations $\left(-45^{\circ}, 0^{\circ}, 45^{\circ}\right.$, and $90^{\circ}$ ), (ii) stable ultra-wideband [defined by the U.S. Federal Communications Commission (FCC) in [38] as a signal with an instantaneous spectral occupancy in excess of $500 \mathrm{MHz}$ or a fractional bandwidth of $20 \%$ or more] antenna performance for all polarization states without additional reconfigurable matching networks, (iii) protection of the PIN diodes without radome by integrating the electronic components inside the cavity, (iv) high radiation efficiency owing to the AFSIW technology, (v) low component count by avoiding additional capacitors/inductors to isolate the DC bias from the RF signals, owing to the dedicated antenna topology with incorporated biasing network and (vi) excellent antenna/platform isolation. In contrast to [8], where a reconfigurable dual-linearly-polarized AFSIW cavity-backed slot antenna is presented, this antenna features dynamic switching between four linear polarization states by an external bias tee and an innovative biasing network that exploits the AFSIW sidewalls to route the required DC bias 
currents to the PIN diodes in the antenna cavity. Moreover, the proposed antenna is directly implemented in medium-density fiberboard (MDF), one of the most commonly used materials to manufacture furniture. In Table II, a comparison to the stateof-the-art proves excellent performance in terms of bandwidth, antenna efficiency and complexity.

\section{Multipolarization ReCOnFIGurable AFSIW CAVITY-BACKED SLOT ANTENNA}

Fig. 1 shows the proposed polarization reconfigurable AFSIW cavity-backed slot antenna. A substrate-independent polarization-reconfigurable AFSIW cavity-backed slot antenna topology, extending the topology in [8], is adopted to realize dynamic reconfiguration between four linear polarizations through four quartets of PIN diodes biased via an external bias tee. The AFSIW topology overcomes the low efficiency and narrow bandwidth of microstrip antennas and preserves the advantages of planar technology, being a cost-effective fabrication, lightweight, and easy integration with other planar circuitry. Moreover, judiciously positioning rows of vias [39] guarantees maximal suppression of surface waves and excellent antenna/platform isolation while, by inserting a well-defined airfilled region, avoiding the disadvantages of the dielectric-filled SIW counterpart, being a performance that strongly depends on the substrate material [40], [41].

The antenna consists of a cavity top plane (Layer A), serving as the antenna aperture, a middle dielectric layer (Layer B), providing an air-filled cavity and support for the SIW sidewalls implemented by tubelets, and a cavity back plane (Layer C). The SIW sidewalls and electric contact between the PCB layers are achieved by tubelets routed through air-holes, present in each layer. The design procedure described in [8] was leveraged to minimize loss and to obtain nearly substrate-independent behavior. As a starting point, a circular air-cavity is created in Layer B. It is fed at its center via a coaxial connector and its radius is dimensioned such that only its fundamental mode,

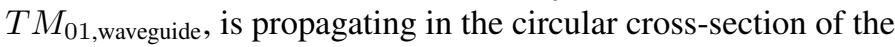
cavity. Next, a double-ring of slots is inserted in the top plane of the cavity to achieve an ultrawide impedance bandwidth. Then, two diametrically positioned shorting tabs are added to split both annular slots in two. In each half-ring radiating

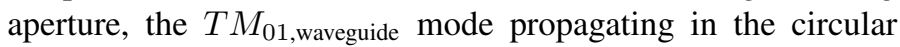
cross-section of the feeding cavity is converted into the $T E_{11 \text {,slot }}$ even mode of that slot. Consequently, a conical beam radiation pattern is produced, as shown in Fig. 2. In particular, the angular location of the shorting tabs defines the direction along which the electric field is linearly polarized, given the symmetry of the structure (for example, the antenna in Fig. 2(a) exhibits $+45^{\circ}$-oriented linear polarization whereas $90^{\circ}$-oriented linear polarization is achieved by the antenna in Fig. 2(b)). Remark that the radiation pattern is not fully omnidirectional in the azimuth plane as the electric field distribution in the apertures is no longer rotationally invariant due to the shorting tabs [8].

To implement reconfiguration between four linear polarizations, the AFSIW antenna topology, shown in the insets of Fig. 2, is adapted to the topology in Fig. 1. Instead of fixing the polarization by the angular locations of the shorting tabs in Layer A, dynamic reconfiguration is achieved by replacing

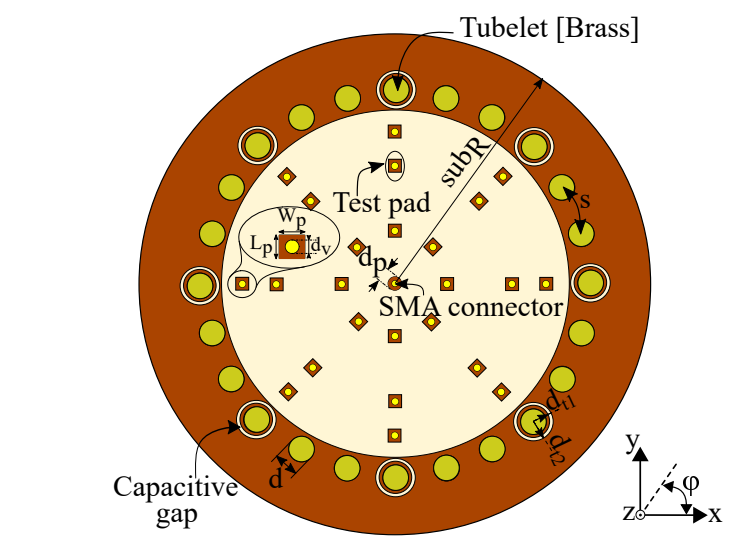

(a)

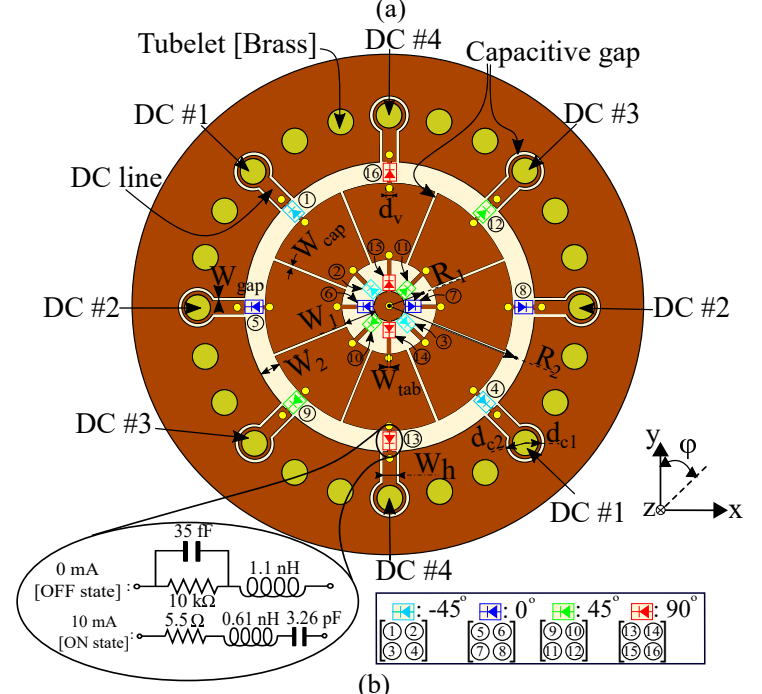

(b)

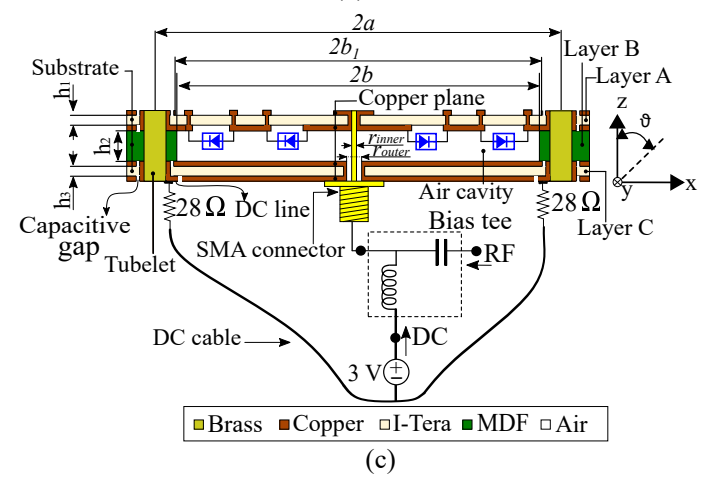

Fig. 1. Multipolarization-reconfigurable AFSIW cavity-backed slot antenna. (a) Front side of Layer A. (b) Back side of Layer A. (c) Cross section view. Optimized dimensions: $s u b_{R}=55.0 \mathrm{~mm}, a=30.5 \mathrm{~mm}, b=b_{1}=27.9 \mathrm{~mm}$, $R_{1}=4.55 \mathrm{~mm}, W_{1}=5.1 \mathrm{~mm}, R_{2}=22.3 \mathrm{~mm}, W_{2}=3.5 \mathrm{~mm}, W_{t a b}=0.2 \mathrm{~mm}$, $W_{\text {cap }}=0.1 \mathrm{~mm}, W_{\text {gap }}=0.15 \mathrm{~mm}, W_{p}=1.5 \mathrm{~mm}, W_{h}=1.8 \mathrm{~mm}$, $L_{p}=1.5 \mathrm{~mm}, d_{v}=1.0 \mathrm{~mm}, h_{1}=h_{3}=0.25 \mathrm{~mm}, h_{2}=4.0 \mathrm{~mm}, d=4.0 \mathrm{~mm}$, $d_{p}=2.5 \mathrm{~mm}, d_{c 1}=d_{t 1}=3.35 \mathrm{~mm}, d_{c 2}=d_{t 2}=3.5 \mathrm{~mm}, s=8.1 \mathrm{~mm}$, $r_{\text {inner }}=1.3 \mathrm{~mm}, r_{\text {outer }}=4.3 \mathrm{~mm}$. Substrate parameters at $5.5 \mathrm{GHz}$ : Layers $\mathrm{A}$ and $\mathrm{C}$ are implemented in I-Tera $\left(\epsilon_{r}=3.43, \tan \delta=0.014\right)$, Layer B is implemented in MDF material $\left(\epsilon_{r}=2.21, \tan \delta=0.06\right)$.

these short-circuits by PIN diodes at carefully selected angular positions [Fig. 1(b)]. Specifically, four quartets of PIN diodes are positioned to switch between the four (linear along $-45^{\circ}, 0^{\circ}$, $+45^{\circ}$ or $90^{\circ}$-direction) polarization states. To dynamically and independently control each quartet of PIN diodes, capacitive gaps are created on Layer A and Layer C [Fig. 1 and Fig. 3], to decouple the DC grounds of the diodes. In addition, the DC lines on Layer A [Fig. 1(b) and Fig. 3(a)] are extended through the AFSIW sidewall vias to the bottom layer of Layer 


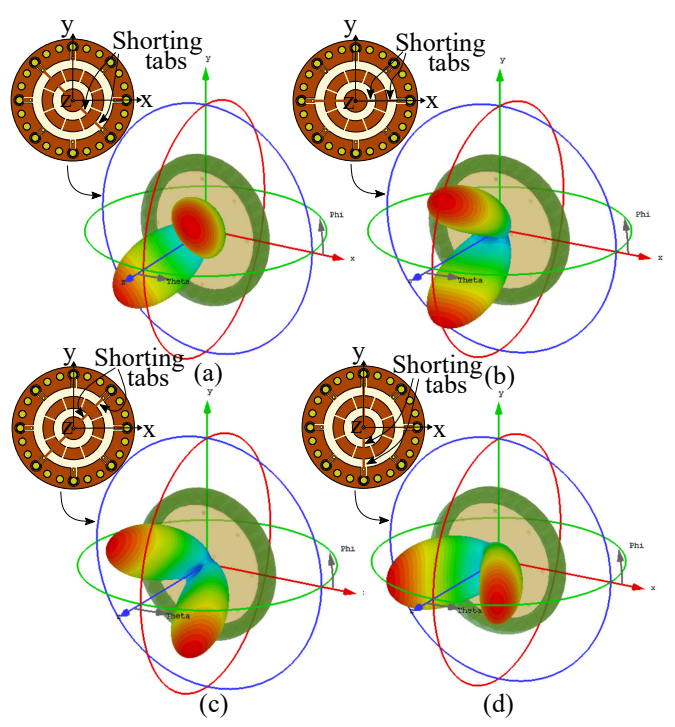

Fig. 2. 3D gain pattern on a linear scale. In all gain patterns, the $\left|E_{\theta}\right|$ is the main component of the electric field $\left(\left|E_{\varphi}\right| \ll\left|E_{\theta}\right|\right)$. (a) Mode\#1, (b) Mode\#2, (c) Mode\#3, (d) Mode\#4.

TABLE I

Polarization STATES By DifFERENT STATUS OF PIN Diodes

\begin{tabular}{l||c||c||c||c}
\hline \multicolumn{1}{c||}{$\begin{array}{c}\text { Diode Color / } \\
\text { Bias Current }\end{array}$} & $\begin{array}{c}\text { Mode\#1 } \\
-45^{\circ}\end{array}$ & $\begin{array}{c}\text { Mode\#2: } \\
0^{\circ}\end{array}$ & $\begin{array}{c}\text { Mode\#3: } \\
45^{\circ}\end{array}$ & $\begin{array}{c}\text { Mode\#4: } \\
90^{\circ}\end{array}$ \\
\hline Cyan / DC\#1 (mA) & ON / 10 & OFF / 0 & OFF / 0 & OFF / 0 \\
Blue / DC\#2 (mA) & OFF / 0 & ON / 10 & OFF / 0 & OFF / 0 \\
Green / DC\#3 (mA) & OFF / 0 & OFF / 0 & ON / 10 & OFF / 0 \\
Red / DC\#4 (mA) & OFF / 0 & OFF / 0 & OFF / 0 & ON / 10 \\
\hline
\end{tabular}

C [Fig. 1(c) and Fig. 3(d)]. In this way, it is possible to route the DC bias current from the front side of the antenna to the backside. By applying a positive polarity at the antenna RF port through the bias tee, while the other polarity is supplied to the DC\#1 pins, and not to the other DC biasing lines [Fig. 1(b) and Fig. 3(d)], the cyan-colored diode quartet $(1,2,3$ and 4$)$ is brought in the ON state and the other diodes are switched OFF, yielding a $45^{\circ}$ linear polarization as in Fig. 2(a). Similarly, when the DC current bias line is switched to any of the other DC biasing lines described in Table I, the desired polarization state switching will be realized as in Fig. 2. Hence, by controlling the ON/OFF status of these four diode quartets, the polarization state can switch between any of the four polarization states shown in Table I and Fig. 2. The current through each diode is set to $10 \mathrm{~mA}$ by adding a $28 \Omega$ series resistor to each DC wire at the back of the antenna. A voltage of $3 \mathrm{~V}$ is supplied through the external bias tee [Fig. 1(c)].

An antenna prototype is fabricated by two standard costeffective manufacturing techniques. First, Layer B is implemented in MDF, by patterning the air cavities and the via holes for the insertion of tubelets by a standard BRM100160 laser cutter [42] [Fig. 3(b)]. Next, the antenna slot plane (Layer A) and antenna back plane (Layer C) are realized on a standard two-layer I-Tera laminate [43] by conventional PCB manufacturing techniques. Note that Layer $\mathrm{C}$ can also be realized on a low-cost dielectric material, such as FR4 [44] without performance degradation, since it is not a part of the antenna cavity. All diodes are soldered on the backside of Layer A before assembly of all layers [Fig. 3(a)]. Here, the concept is demonstrated using MACOM's MA4AGBLP912 PIN diodes [45]. Their equivalent circuit model for each state [8] is depicted in Fig. 1(b). This approach provides robust and

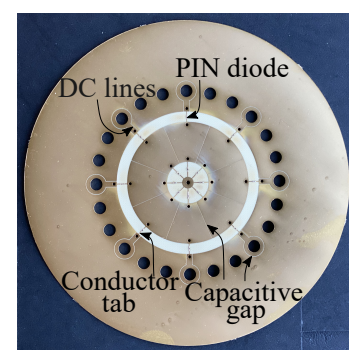

(a)

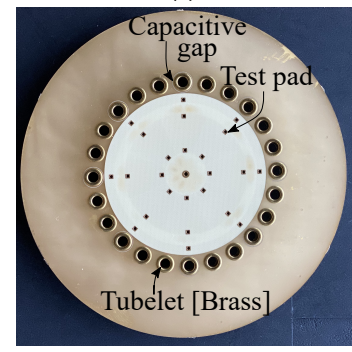

(c)

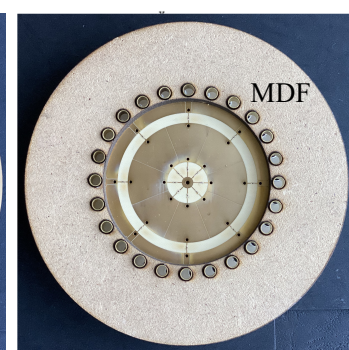

(b)

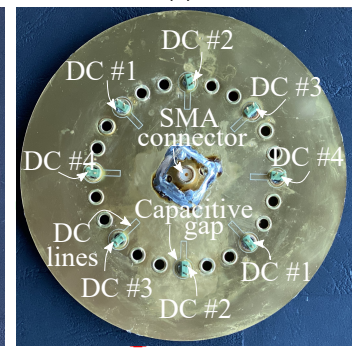

(d)
Fig. 3. Picture of the fabricated multipolarization-reconfigurable AFSIW cavitybacked slot antenna. (a) Back side of Layer A. (b) Integrated Layer A inside Layer B. (c) Top view of assembled prototype. (d) Back side of Layer C.

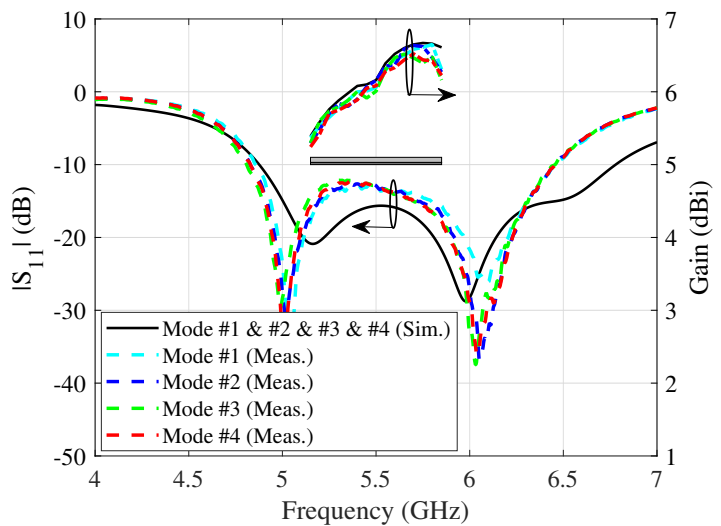

Fig. 4. Measured and simulated $\left|S_{11}\right|$ w.r.t. $50 \Omega$ and gain versus frequency for four modes.

invisible integration of all electronic components inside the antenna cavity, while ensuring protection against environmental influences. To verify the PIN diode operation after assembly and to control the biasing states, small vias are inserted in the copper test pads and biasing pads in Layer A [Fig. 1(a)]. Finally, the three layers are joined by punching conductive tubelets through the appropriate holes [Fig. 1(c) and Fig. 3(c)].

\section{iII. Simulation and Measurement Results}

Fig. 3 shows the fabricated reconfigurable antenna. The input reflection coefficients are measured by a Keysight N5242A PNA-X Network Analyzer. The radiation performance is validated in an anechoic chamber by an NSI-MI spherical near-field antenna measurement system. A Keithley 2450 sourcemeter and an SHF Communication Technologies, SHF BT45A bias tee provide DC biasing, according to Table I.

The simulated and measured magnitudes of the input reflection coefficients in the various states are shown in Fig. 4. They are in good agreement, considering the various sources of fabrication and component tolerances. In all states, the antenna is matched to $Z_{0}=50 \Omega$ from $4.85 \mathrm{GHz}$ to $6.45 \mathrm{GHz}$, yielding a $-10 \mathrm{~dB}$ impedance bandwidth of $1.6 \mathrm{GHz}$, or $29 \%$, covering the entire [5.15-5.85] GHz U-NII radio band. 


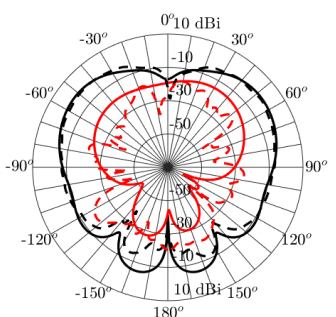

(a)

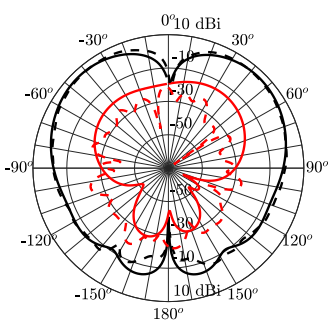

(e)

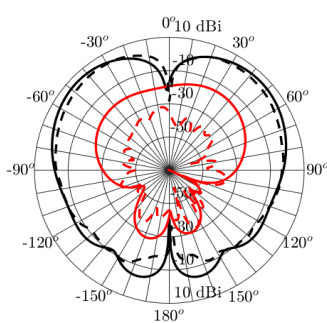

(b)

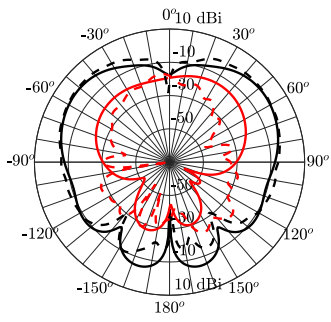

(f)

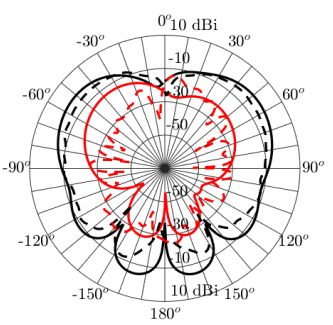

(c)

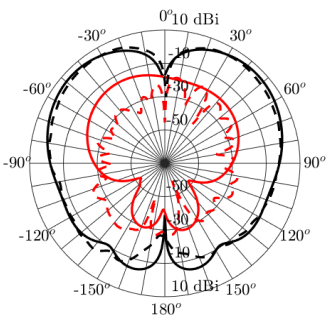

(g)

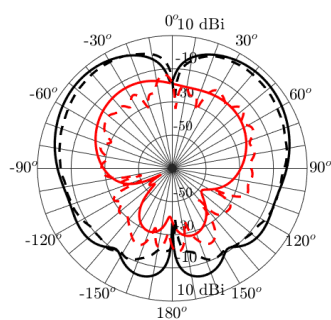

(d)

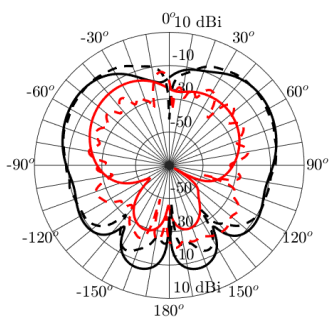

(h)

Fig. 5. Measured and simulated radiation patterns at $5.5 \mathrm{GHz}$. Mode\#1: (a) $\phi=-45^{\circ}$-plane and (b) $\phi=45^{\circ}$-plane. Mode $\# 2$ : (c) $\phi=0^{\circ}$-plane and (d) $\phi=90^{\circ}$ plane. Mode\#3: (e) $\phi=-45^{\circ}$-plane and (f) $\phi=45^{\circ}$-plane. Mode\#4: (g) $\phi=0^{\circ}$-plane and (h) $\phi=90^{\circ}$-plane.

TABLE II

Performance Comparison of Multiple Polarization Reconfigurable Antennas

\begin{tabular}{|c|c|c|c|c|c|c|c|c|c|}
\hline Ref. & [8] & [31] & [46] & [47] & [48] & [49] & [50] & [51] & This work \\
\hline Number of polarization states & 2 & 6 & 3 & 6 & 3 & 3 & 4 & 6 & 4 \\
\hline Overlapped bandwidth (\%) & 16.3 & $5 * *$ & 25.9 & 2 & $3.4 * *$ & 3.08 & 1.2 & 1.1 & $>29$ \\
\hline Number of antenna elements & 1 & 1 & 1 & 1 & 4 & 4 & 1 & 1 & 1 \\
\hline Overlapped peak gain $(\mathrm{dBi})$ & 7.3 & 3.52 & 6.7 & 3 & 10.6 & 10.75 & $4.7 * *$ & $7 * *$ & 6.5 \\
\hline Radiation efficiency (\%) & $>95$ & $46^{*}$ & 59 & $60 *$ & - & $>54.5$ & $>39.9 * *$ & - & $>70$ \\
\hline Number of ports & 1 & 1 & 1 & 1 & 1 & 1 & 1 & 2 & 1 \\
\hline Antenna size $\left(\lambda_{0}\right)$ & $1.1 \times 1.1$ & $0.52 \times 0.52$ & $1.1 \times 1.1$ & $0.2 \times 0.2$ & $3.1 \times 2.7$ & $2.9 \times 2.3$ & $0.9 \times 0.8$ & $0.75 \times 0.75$ & $1.1 \times 1.1$ \\
\hline
\end{tabular}

The radiation patterns are simulated and measured at different frequencies for each of the four polarization modes. Fig. 5 depicts a good agreement between the simulated and measured radiation patterns at $5.5 \mathrm{GHz}$ for $-45^{\circ}, 0^{\circ}, 45^{\circ}$, and $90^{\circ}$ linear polarizations, with a boresight gain of $6.05 \mathrm{dBi}$ at $(\theta, \phi)=\left(44^{\circ}, 45^{\circ}\right), 6.16 \mathrm{dBi}$ at $(\theta, \phi)=\left(44^{\circ}, 90^{\circ}\right), 6.01 \mathrm{dBi}$ at $(\theta, \phi)=\left(44^{\circ},-45^{\circ}\right)$ and $6.08 \mathrm{dBi}$ at $(\theta, \phi)=\left(44^{\circ}, 0^{\circ}\right)$, respectively. The difference in maximum gain between both orthogonal planes is $5.6 \mathrm{~dB}$ for Mode\#1, $6.6 \mathrm{~dB}$ for Mode\#2, 5.8 $\mathrm{dB}$ for Mode\#3 and $5.7 \mathrm{~dB}$ for Mode\#4. A 3-dB beamwidth larger than $45^{\circ}$ can be observed at each polarization mode, while maintaining a front-to-back ratio higher than $10.5 \mathrm{~dB}$. In addition, the measured mean cross-polarization ratio within that 3-dB beamwidth remains higher than $17 \mathrm{~dB}$. Furthermore, linear polarization is achieved in the $\phi=45^{\circ}, \phi=90^{\circ}, \phi=-$ $45^{\circ}$ and $\phi=0^{\circ}$ planes with the axial ratio over $40 \mathrm{~dB}$ for each polarization mode. Finally, a total antenna efficiency of $72 \%$, $74 \%, 72 \%$ and $73 \%$ is measured for $-45^{\circ}, 0^{\circ}, 45^{\circ}$, and $90^{\circ}$ linear polarizations, respectively. In Fig. 4, the simulated and measured maximum gain across the operating band agree well, with measured realized gains between $5.2 \mathrm{dBi}$ and $6.5 \mathrm{dBi}$. The measured total antenna efficiency varies between $70 \%$ to $80 \%$, which is slightly lower than the simulated total efficiency due to additional losses in the diodes.

Table II compares the proposed AFSIW cavity-backed annular slot antenna with multipolarization reconfiguration to the current state-of-the-art in terms of antenna performance and footprint. It can be seen that the presented antenna fea- tures excellent performance with respect to the other designs, especially when considering the combination of impedance bandwidth and radiation efficiency. Furthermore, owing to its substrate-independent performance, our antenna topology maintains stable performance, even after direct implementation in everyday surfaces, in contrast to other state-of-the-art designs. When considering number of polarization states, the antenna topologies in [31], [47] and [51] provide remarkable performance in a compact footprint. Yet, they do not exhibit ultrawideband performance and their broadside radiation pattern is less appropriate for smart surfaces.

\section{CONCLUSiON}

A very wide bandwidth, single-feed multipolarization reconfigurable AFSIW cavity-backed slot antenna is presented for IoT applications in the [5.15-5.85] GHz U-NII radio band. To the authors' best knowledge, this is the first low-profile, lowcost, stable, broadband multipolarization reconfigurable AFSIW antenna in commonly available surface materials that facilitates easy surface integration by placing PIN diodes inside the AFSIW antenna cavity. In addition, a dedicated biasing network is incorporated to route the DC biasing current between the top and bottom cavity plane through the AFSIW sidewalls. Measurements prove that the antenna exhibits a front-to-back ratio of $10.5 \mathrm{~dB}$, a total antenna efficiency higher than $70 \%$, and $5.2 \mathrm{dBi}$ gain in the frequency range [4.85-6.45] $\mathrm{GHz}$ for each polarization state. Finally, an axial ratio of $40 \mathrm{~dB}$ for $-45^{\circ}$, $0^{\circ}, 45^{\circ}$, and $90^{\circ}$ linear polarization is reported in the $\phi=45^{\circ}$, $\phi=90^{\circ}, \phi=-45^{\circ}$ and $\phi=0^{\circ}$ planes, respectively. 


\section{REFERENCES}

[1] G. Kortuem, F. Kawsar, V. Sundramoorthy, and D. Fitton, "Smart objects as building blocks for the Internet of Things," IEEE Internet Comput., vol. 14, no. 1, pp. 44-51, Jan. 2010.

[2] L. Roselli et al., "Smart surfaces: Large area electronics systems for internet of things enabled by energy harvesting," Proc. IEEE, vol. 102, no. 11 , pp. 1723-1746, Nov. 2014

[3] O. Caytan et al., "Half-mode substrate-integrated-waveguide cavitybacked slot antenna on cork substrate," IEEE Antennas Wireless Propag. Lett., vol. 15, pp. 162-165, 2016.

[4] S. Lemey et al., "Threefold rotationally symmetric SIW antenna array for ultra-short-range MIMO communication," IEEE Trans. Antennas Propag., vol. 64, no. 5, pp. 1689-1699, May 2016.

[5] N. J. McEwan, R. A. Abd-Alhameed, E. M. Ibrahim, P. S. Excell, and J. G. Gardiner, "A new design of horizontally polarized and dual-polarized uniplanar conical beam antennas for HIPERLAN," IEEE Trans. Antennas Propag., vol. 51, no. 2, pp. 229-237, Feb. 2003.

[6] Z.-C. Hao, H.-H. Wang, and W. Hong, "A novel planar reconfigurable monopulse antenna for indoor smart wireless access points' application,' IEEE Trans. Antennas Propag., vol. 64, no. 4, pp. 1250-1261, Apr. 2016.

[7] H. F. Khalili et al., "Biodegradable dual semicircular patch antenna tile for smart floors," IEEE Antennas Wireless Propag. Lett., vol. 18, no. 2, pp. 368-372, Feb. 2019.

[8] K. Y. Kapusuz, S. Lemey, A. Petrocchi, P. Demeester, D. Schreurs, and H. Rogier, "Polarization reconfigurable air-filled substrate integrated waveguide cavity-backed slot antenna," IEEE Access, vol. 7, pp. 102628 $102643,2019$.

[9] M. Boti, L. Dussopt, and J.-M. Laheurte, "Circularly polarised antenna with switchable polarisation sense," Electron. Lett., vol. 36, no. 18, pp. 1518-1519, Aug. 2000.

[10] F. Yang and Y. Rahmat-Samii, "A reconfigurable patch antenna using switchable slots for circular polarization diversity," IEEE Microw. Wireless Compon. Lett., vol. 12, no. 3, pp. 96-98, Mar. 2002.

[11] J.-F. Wu and J.-S. Row, "Broadband circularly-polarised microstrip antenna with switchable polarisation sense," Electron. Lett., vol. 42, no. 24, pp. 1374-1375, Nov. 2006.

[12] W. Lin and H. Wong, "Wideband circular polarization reconfigurable antenna," IEEE Trans. Antennas Propag., vol. 63, no. 12, pp. 5938-5944, Dec. 2015.

[13] —, "Wideband circular-polarization reconfigurable antenna with Lshaped feeding probes," IEEE Antennas Wireless Propag. Lett., vol. 16, pp. 2114-2117, 2017.

[14] H. H. Tran, N. Nguyen-Trong, T. K. Nguyen, and A. Abbosh, "Bandwidth enhancement utilizing bias circuit as parasitic elements in a reconfigurable circularly polarized antenna," IEEE Antennas Wireless Propag. Lett. vol. 17, no. 8, pp. 1533-1537, Aug. 2018.

[15] S. Gao, H. Lin, L. Ge, and D. Zhang, "A magneto-electric dipole antenna with switchable circular polarization," IEEE Access, vol. 7, pp. 40013 40018,2019

[16] J. Hu, Z.-C. Hao, K. Fan, and Z. Guo, "A bidirectional same sense circularly polarized endfire antenna array with polarization reconfigurability," IEEE Trans. Antennas Propag., vol. 67, no. 11, pp. 7150-7155, Nov. 2019.

[17] Y. Li, Z. Zhang, W. Chen, and Z. Feng, "Polarization reconfigurable slot antenna with a novel compact CPW-to-slotline transition for WLAN application," IEEE Antennas Wireless Propag Lett, vol. 9, pp. 252-255, 2010.

[18] P.-Y. Qin, A. R. Weily, Y. J. Guo, and J.-H. Liang, "Polarization reconfigurable U-slot patch antenna," IEEE Trans. Antennas Propag., vol. 58, no. 10 , pp. $3383-3388$, Oct. 2010.

[19] W. Lin and H. Wong, "Polarization reconfigurable aperture-fed patch antenna and array," IEEE Access, vol. 4, pp. 1510-1517, 2016.

[20] J.-S. Row, W.-L. Liu, and T.-R. Chen, "Circular polarization and polarization reconfigurable designs for annular slot antennas," IEEE Trans. Antennas Propag., vol. 60, no. 12, pp. 5998-6002, Dec. 2012.

[21] J.-S. Row and C.-J. Shih, "Polarization-diversity ring slot antenna with frequency agility," IEEE Trans. Antennas Propag., vol. 60, no. 8, pp. 3953-3957, Aug. 2012.

[22] C.-Y.-D. Sim, Y.-J. Liao, and H.-L. Lin, "Polarization reconfigurable eccentric annular ring slot antenna design," IEEE Trans. Antennas Propag., vol. 63, no. 9, pp. 4152-4155, Sep. 2015.

[23] K. X. Wang and H. Wong, "A reconfigurable CP/LP antenna with crossprobe feed," IEEE Antennas Wireless Propag. Lett., vol. 16, pp. 669-672, 2017.
[24] H. Wong, W. Lin, L. Huitema, and E. Arnaud, "Multi-polarization reconfigurable antenna for wireless biomedical system," IEEE Trans. Biomed. Circuits Syst., vol. 11, no. 3, pp. 652-660, Jun. 2017.

[25] H. H. Tran, N. Nguyen-Trong, T. T. Le, A. M. Abbosh, and H. C. Park, "Low-profile wideband high-gain reconfigurable antenna with quadpolarization diversity," IEEE Trans. Antennas Propag., vol. 66, no. 7, pp. 3741-3746, Jul. 2018.

[26] Y. Sung, T. Jang, and Y.-S. Kim, "A reconfigurable microstrip antenna for switchable polarization," IEEE Microw. Wireless Compon. Lett., vol. 14, no. 11 , pp. 534-536, Nov. 2004

[27] W. Lin and H. Wong, "Polarization reconfigurable wheel-shaped antenna with conical-beam radiation pattern," IEEE Trans. Antennas Propag., vol. 63, no. 2, pp. 491-499, Feb. 2015.

[28] S.-L. Chen, F. Wei, P.-Y. Qin, J. Guo, and X. Chen, "A multi-linear polarization reconfigurable unidirectional patch antenna," IEEE Trans. Antennas Propag., vol. 65, no. 8, pp. 4299-4304, Aug. 2017.

[29] H. H. Tran, N. Nguyen-Trong, T. T. Le, and H. C. Park, "Wideband and multipolarization reconfigurable crossed bowtie dipole antenna," IEEE Trans. Antennas Propag., vol. 65, no. 12, pp. 6968-6975, Dec. 2017.

[30] Y. Yang, R. B. V. B. Simorangkir, X. Zhu, K. Esselle, and Q. Xue, "A novel boresight and conical pattern reconfigurable antenna with the diversity of $360^{\circ}$ polarization scanning," IEEE Trans. Antennas Propag., vol. 65, no. 11 , pp. 5747-5756, Nov. 2017.

[31] L. Chang, W. Lai, J. Cheng, and C. Hsue, "A symmetrical reconfigurable multipolarization circular patch antenna," IEEE Antennas and Wireless Propag. Lett., vol. 13, pp. 87-90, 2014.

[32] Z. Chen, H. Wong, and J. Kelly, "A polarization-reconfigurable glass dielectric resonator antenna using liquid metal," IEEE Trans. Antennas Propag., vol. 67, no. 5, pp. 3427-3432, May 2019.

[33] H. Sun and S. Sun, "A novel reconfigurable feeding network for quadpolarization-agile antenna design," IEEE Trans. Antennas Propag., vol. 64, no. 1, pp. 311-316, Jan. 2016

[34] W. Lin and H. Wong, "Multipolarization-reconfigurable circular patch antenna with L-shaped probes," IEEE Antennas and Wireless Propag. Lett., vol. 16, pp. 1549-1552, 2017.

[35] Y. J. Guo, P.-Y. Qin, S.-L. Chen, W. Lin, and R. W. Ziolkowski, "Advances in reconfigurable antenna systems facilitated by innovative technologies," IEEE Access, vol. 6, pp. 5780-5794, 2018.

[36] J.-S. Row and Y.-H. Wei, "Wideband reconfigurable crossed-dipole antenna with quad-polarization diversity," IEEE Trans. Antennas Propag., vol. 66, no. 4, pp. 2090-2094, Apr. 2018.

[37] Z. Chen, H. Wong, and Y. Liu, "A polarizer integrated dielectric resonator antenna for polarization reconfigurability," IEEE Trans. Antennas Propag., vol. 67, no. 4, pp. 2723-2728, Apr. 2019.

[38] "Revision of part 15 of the commission's rules regarding ultra-wideband transmission systems," Federal Commun. Commission, Washington, DC, USA, Tech. Rep. ET Docket 98-153, Apr. 2002.

[39] A. Kumar, "Wideband circular cavity-backed slot antenna with conical radiation patterns," Microw. and Opt. Technol. Lett., vol. 62, no. 6, pp. 2390-2397, Jun. 2020.

[40] K. Y. Kapusuz, S. Lemey, and H. Rogier, "Substrate-independent microwave components in substrate integrated waveguide technology for high-performance smart surfaces," IEEE Trans. Microw. Theory Techn., vol. 66, no. 6, pp. 3036-3047, Jun. 2018.

[41] F. Parment, A. Ghiotto, T. Vuong, J. Duchamp, and K. Wu, "Air-filled substrate integrated waveguide for low loss and high-power handling millimeter-wave substrate integrated circuits," IEEE Trans. Microw. Theory Techn., vol. 63, no. 4, pp. 1228-1238, Apr. 2015.

[42] Available: https://static.webshopapp.com/shops/118762/files/188871857/brmstandard-user-manual-en-v1.pdf on: October 18, 2020.

[43] Available: http://www.isola-group.com/wp-content/uploads/ITera-MT40RF-Laminte-and-Prepreg-Data-Sheet-0417.pdf on: October 18, 2020.

[44] Available: https://www.farnell.com/datasheets/1644697.pdf on: October 18, 2020.

[45] Available: https://cdn.macom.com/datasheets/MA4AGBLP912.pdf on: October 18, 2020

[46] W.-J. Sun, W.-W. Yang, L. Guo, W. Qin, and J.-X. Chen, "A circularly polarized dielectric resonator antenna and its reconfigurable design," IEEE Antennas Wireless Propag. Lett., vol. 19, no. 7, pp. 1088-1092, Jul. 2020.

[47] N. Nguyen-Trong, A. Piotrowski, L. Hall, and C. Fumeaux, "A frequency and polarization-reconfigurable circular cavity antenna," IEEE Antennas Wireless Propag. Lett., vol. 16, pp. 999-1002, 2017.

[48] Z.-C. Hao, K.-K. Fan, and H. Wang, "A planar polarization-reconfigurable antenna," IEEE Trans. Antennas Propag., vol. 65, no. 4, pp. 1624-1632, Apr. 2017 
[49] J. Hu, Z. C. Hao, and Z. W. Miao, "Design and implementation of a planar polarization-reconfigurable antenna," IEEE Antennas Wireless Propag. Lett., vol. 16, pp. 1557-1560, 2017.

[50] L. Ge, Y. Li, J. Wang, and C.-Y.-D. Sim, "A low-profile reconfigurable cavity-backed slot antenna with frequency, polarization, and radiation pattern agility," IEEE Trans. Antenna Propag., vol. 65, no. 5, pp. 2182 2189, May 2017.

[51] H. Lee, Y. Sung, C. T. M. Wu, and T. Itoh, "Dual-band and polarization flexible cavity antenna based on substrate integrated waveguide," IEEE Antennas Wireless Propag. Lett., vol. 15, pp. 488-491, 2016. 